\title{
A percepção dos enfermeiros da atenção básica sobre a integralidade na saúde
}

\author{
The nurses' perception of the basic attention on health integrality \\ La percepción de las enfermeras de atención primaria sobre la integralidad en salud \\ Suyane de Souza Lemos ${ }^{1 *}$, Wilza Carla Spiri ${ }^{1}$, Regina Stella Spagnuolo ${ }^{1}$.
}

\begin{abstract}
RESUMO
Objetivo: Conhecer a percepção dos enfermeiros da atenção básica sobre a integralidade na saúde. Métodos: Optou-se pela abordagem qualitativa, na vertente da fenomenologia. A saturação teórica se configurou, por meio da análise de vinte e duas entrevistas, sendo os sujeitos enfermeiros da atenção básica. Resultados: Emergiram dos sujeitos elementos desafiadores como modelo biomédico centrado, falta de infraestrutura, alta demanda, dificuldade de comunicação entre os serviços para a efetivação da integralidade, por outro lado, as fortalezas são a própria estratégia saúde da família, o apoio matricial do Núcleo de Apoio à Saúde da Família na produção do cuidado, a valorização do trabalho em equipe interdisciplinar, necessidade de maiores investimentos na educação permanente na saúde. Conclusão: Assim, o estudo permitiu reflexões acerca da integralidade, a atenção à saúde deve ser acolhedora, humanizada, contextualizada pelo olhar para o território, ou seja, o contexto que o usuário está inserido como forma de planejar intervenções ao mesmo.
\end{abstract}

Palavras-chave: Atenção primária à saúde, Enfermagem em saúde comunitária, Integralidade em saúde, Educação em enfermagem, Políticas de saúde.

\section{ABSTRACT}

Objective: To know the nurses' perception in primary care about integrality in health. Methods: We opted for the qualitative approach in the field of phenomenology. Theoretical saturation was configured by analyzing twenty-two interviews, where the subjects were nurses working in primary care. Results: Challenging elements emerged from the subjects, such as a centered biomedical model, lack of infrastructure, high demand, difficulty of communication among services for the effectiveness of integrality. Conversely, the strengths are the Family Health Strategy itself, the matrix support of the Family Health Support Nucleus in the production of care, the valorization of interdisciplinary teamwork, as well as the need for higher investments in continuing health education. Conclusion: Accordingly, the study allowed us to make reflections on integrality, where health care should be welcoming, humanized, contextualized by looking at the territory, i.e., the context in which the user is inserted, as a way of planning its interventions.

Keywords: Primary health care, Community health nursing, Integrality in health, Nursing education, Health policies.

\section{RESUMEN}

Objetivo: Conocer la percepción de enfermeros de atención primaria sobre la integralidad en salud. Métodos: Optamos por el enfoque cualitativo en el ámbito de la fenomenología. La saturación teórica se configuró mediante el análisis de veintidós entrevistas, donde los sujetos eran enfermeros de atención primaria. Resultados: De los sujetos, surgieron elementos desafiantes, como modelo biomédico centrado, falta de infraestructura, alta demanda, dificultad de comunicación entre los servicios para la efectividad de la integralidad. En contraste, las fortalezas son la propia Estrategia de Salud Familiar, el apoyo matricial del Núcleo de Apoyo a la Salud de la Familia en la producción de atención, la valorización del trabajo en equipo interdisciplinario y la necesidad de mayores inversiones en educación continua en salud. Conclusión: Así, el estudio permitió reflexiones sobre la integralidad, donde la atención de la salud debe ser acogedora, humanizada, contextualizada mediante la mirada al territorio, es decir, el contexto en el que se inserta el usuario como forma de planificar sus intervenciones.

Palabras clave: Atención primaria de salud, Enfermería en salud comunitária, Integralidad en salud, Educación en enfermeira, Políticas de salud.

${ }^{1}$ Universidade Estadual Paulista Júlio de Mesquita Filho. Botucatu - SP. *E-mail: suyane.lemos.ss@gmail.com SUBMETIDO EM: 5/2020 ACEITO EM: 6/2020 PUBLICADO EM: 10/2020 


\section{INTRODUÇÃO}

Os sistemas de atenção à saúde são respostas sociais às necessidades de saúde da população, sendo necessário reduzir desigualdades, fortalecer a regionalização e ampliar o conceito de saúde (LOUVISON MCP, 2019). Lima SGG, et al. (2019) relata que com o aumento significativo da importância política e econômica da atenção à saúde no Brasil houve a expansão dos serviços de saúde, acompanhada da oferta de novas tecnologias e do desenvolvimento de modelos assistenciais para intervenções na área da saúde comparando com a realidade mundial.

Mendes EV (2010) reflete sobre a crise dos sistemas de saúde contemporâneos que se explica pela incoerência entre uma situação de saúde com predomínio forte de condições crônicas e uma resposta de atenção à saúde por meio de sistemas fragmentados e voltados, principalmente, para as condições agudas. O que mostra a necessidade de repensar a forma que estamos abordando a nossa população nos serviços de saúde.

A implantação e a consolidação do Sistema Único de Saúde (SUS) desafiam os profissionais e os gestores na condução do sistema como um todo (BRASIL, 1988). Os preceitos básicos do SUS incluem a universalidade, integralidade e equidade no acesso à atenção em saúde, modelo assistencial com ênfase na integralidade das ações, direito à informação, controle social, regionalização e hierarquização das ações (BRASIL, 1990). Neste contexto do SUS, a atenção básica é o contato preferencial dos usuários com os sistemas de saúde, orienta-se pelos princípios da universalidade, da acessibilidade e da coordenação do cuidado, do vínculo e da continuidade, da integralidade, da responsabilização, da humanização, da equidade e da participação social (BRASIL, 2006).

Medeiros AC, et al. (2016) afirma que a integralidade na atenção à saúde é definida como um princípio do SUS, existindo várias políticas que orientam a implementação de ações que respondam às demandas e necessidades da população, nos diversos níveis de atenção e complexidade, nas diferentes abordagens do processo saúde-doença e nas distintas dimensões do ser cuidado (MEDEIROS AC E SIQUEIRA HCH e ZAMBERLAN C, et al., 2016).

Fica evidente a pluralidade de significados da integralidade e suas aplicações no âmbito da saúde. $O$ trabalho se justifica pela necessidade de levantar informações acerca da integralidade para gerar informações que subsidiem a tomada de decisões. Optou-se por focar o olhar no objeto deste estudo: experiência do enfermeiro da atenção básica sobre a integralidade na saúde. A pergunta que norteou a pesquisa foi: Qual a percepção dos enfermeiros da atenção básica sobre a integralidade na saúde? O objetivo do presente estudo foi conhecer a percepção dos enfermeiros da atenção básica sobre a integralidade na saúde.

\section{MÉTODOS}

No desenvolvimento deste estudo a opção pela metodologia qualitativa objetivou a compreensão dos enfermeiros da atenção básica sobre a integralidade na saúde. Entre os principais referenciais utilizados nas pesquisas qualitativas, a autora optou pela fenomenologia.

Merleau PM (2006) considera que a compreensão de significados dos sujeitos que vivenciam determinada situação pode ser melhor apreendida em abordagem qualitativa de pesquisa, recorro à fenomenologia como vertente metodológica. Fizeram parte de todo o estudo vinte e dois enfermeiros da atenção básica do município de São José do Rio Preto que atenderam os critérios de elegibilidade e suficientes para responderem o objetivo da pesquisa (saturação teórica).

Os critérios de elegibilidade foram enfermeiros que atuam, no mínimo, há um ano na saúde e que concordaram em participar da pesquisa com a garantia do anonimato e assinatura do Termo de Consentimento Livre e Esclarecido. Os dados foram coletados a partir de entrevistas norteadas pela seguinte questão: Conte-me sua experiência com a integralidade na saúde? Como o enfermeiro percebe a integralidade na saúde? Quais as ações necessárias para uma ação mais efetiva neste cenário? As entrevistas foram realizadas pelo pesquisador de novembro de 2013 a janeiro de 2014 segundo a conveniência dos sujeitos e audio-gravadas com tempo médio de duração de doze minutos. Cada participante recebeu a sigla "I-1-XXII-22. 
Como se trata de pesquisa envolvendo seres humanos, o projeto foi submetido à aprovação do Comitê de Ética em Pesquisa de acordo com a resolução do Conselho Nacional de Saúde (CNS) com parecer n³49.767. $O$ estudo é resultado de pesquisa de mestrado.

A análise de dados seguiu o referencial da fenomenologia defendido por Sadala MLA (2004), segundo a estrutura do fenômeno incluindo as etapas de análise ideográfica (análise individual dos depoimentos), análise nomotética (análise da globalidade dos depoimentos) e síntese (compreensão do fenômeno pelas convergências, divergências e idiossincrasias).

\section{RESULTADOS E DISCUSSÃO}

A fim de representar alguns temas desvelados na pesquisa, realizamos o mapa conceitual (Figura 1) abaixo.

Figura 1 - Mapa conceitual dos temas da Compreensão da Integralidade das Unidades Básicas de Saúde, n=22.

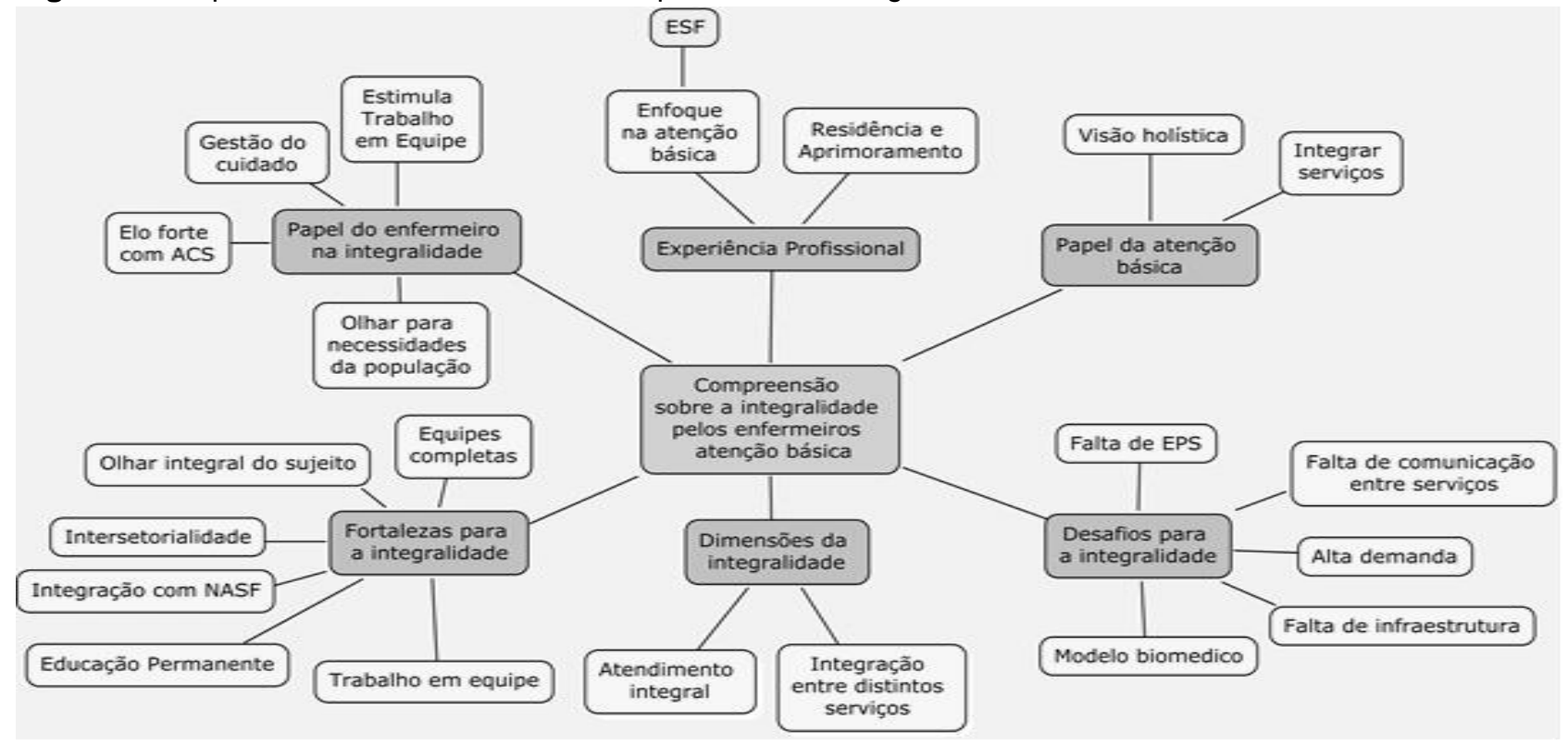

Fonte: LEMOS SS, et al., 2020

Das perspectivas dos sujeitos, emergiram os temas: experiência profissional, papel do enfermeiro na integralidade, papel da atenção básica, dimensões da integralidade, desafios para a integralidade, fortalezas para a integralidade.

Desafios como modelo biomédico, falta de infraestrutura, alta demanda, dificuldade de comunicação entre os serviços foram apontados como elementos desafiadores para a implementação da integralidade, porém as fortalezas também foram fortemente expressadas pelos sujeitos do estudo como valorização do trabalho em equipe interdisciplinar, maiores investimentos na educação permanente na saúde como eixo formador constante dos sujeitos. Envolve também a humanização, acolhimento, gestão do cuidado com foco nas necessidades do sujeito, o apoio matricial do NASF na produção do cuidado são estratégias que envolvem a prática integral.

O tema "Experiência profissional" revela a perspectiva dos sujeitos do estudo que mostraram experiência que permite o planejamento das ações com foco preventivo ampliando a visão integral, além de oportunidades de cursar residência e aprimoramento nesta área. O trabalho na atenção básica também propicia realizar consulta de enfermagem, participar de reuniões, ser gestor do cuidado, ser responsável, conforme expressam as falas:

“(...) na atenção básica (...) eu comecei a aprender de tudo, a fazer consulta de enfermagem (...) a participar de reunião, a atender paciente, a ser chefe, a ser responsável, responsável técnica." (XVI,1) 
Hino $P$, et al. (2019) relata que o enfermeiro tem demonstrado dedicação nas ações propostas pelo Ministério da Saúde. Atividades de planejamento, organização, implementação e avaliação das ações, priorizando ações de promoção e prevenção na saúde, consulta de enfermagem, são atribuições que o enfermeiro vem desenvolvendo no âmbito da saúde da família. Magalhaes MGM e Alvin NAT (2013) citou a incorporação de um conjunto de atividades assistenciais, gerenciais, educativas e de pesquisa de acordo com a política vigente do país. A qualidade da relação enfermeiro- usuário/família, que inclui o acolhimento, configura-se elemento fundamental no atendimento integral e humano às necessidades da população.

Bousquat $A$, et al. (2019), refere que ao que se refere à Residência Multiprofissional, o objetivo é formar competências para o trabalho no SUS, com oportunidades para que propiciem ações significativas em consonância com os princípios e diretrizes do SUS, articulando os conhecimentos da formação inicial.

Oliveira IC, et al. (2018) e Machado MTC, et al. (2015) trazem que a compreensão da integralidade precisa ser desenvolvida durante toda a formação por meio de práticas pedagógicas na atenção básica que favoreçam a aproximação entre o processo formativo e as necessidades dos usuários dos serviços de saúde. Para tanto, Silva MVS, et al. (2017) e Yamamoto TS (2016) afirmam que a educação precisa ser também integral e interdisciplinar, com base em referenciais críticos e reflexivos, desenvolvendo assim competências e habilidades para ações voltadas para o ser humano na sua subjetividade.

O tema "Papel do enfermeiro na integralidade" revela que as atribuições dos enfermeiros colaboram para fortalecer a integralidade na atenção básica.

“(..) vamos até a casa do paciente (...) a gente tenta ser uma equipe multiprofissional, orientando (...) pra manter este vínculo de integralidade (...) ter um acolhimento maior com a população." (IX, 2)

Gomide MFS, et al. (2018) traz que o acolhimento não é uma prática isolada, mas um conjunto de atitudes para atender as necessidades do outro com postura proativa e que envolva a equipe na busca de maximizar as possibilidades de resoluções compartilhando saberes e práticas. Lanzoni GMM e Meirelles BHS (2013) comente que o enfermeiro, como um membro da equipe multiprofissional, deve lutar pelos valores preconizados pelo SUS, bem como a concretização da integralidade na rede básica de saúde. O enfermeiro coordena a equipe, valoriza o planejamento das ações, desenvolve a gestão do cuidado, faz a mediação das ações dos agentes comunitários de saúde, procura conhecer o território de abrangência da unidade de saúde conforme elencado abaixo nas falas:

"(...) o enfermeiro é fundamental (...) na parte do planejamento das ações. (...) de planejar uma ação integral, de discutir as ações, conduzir o trabalho de planejamento" (XV, III).

Lanzoni GMM e Meirelles BHS (2013) relatam ainda que o enfermeiro tem potencial como integrante da equipe para operacionalizar a integralidade procurando responder aos dilemas vivenciados no trabalho coletivo e em equipe. Costa NR (2016), comenta que a interação enfermeira e comunidade com foco na população caminham para resolubilidade e qualidade do atendimento, além de promover a formação de vínculo, promoção da saúde e prevenção de agravos, bem como a autonomia e a corresponsabilidade para a integralidade das ações.

O enfermeiro é observador e investigador das características do paciente, faz análise do contexto que o indivíduo está inserido que vai além da doença e tem um olhar sensível social e ampliado para diversos aspectos como podemos ver abaixo:

"(...) o enfermeiro é meio que uma peça central nesta situação toda (...) tem a percepção do cuidar (...)” (XI, 2).

Facchini LA, et al. (2018) demonstra a necessidade de criação e manutenção de espaços de Educação Permanente em Saúde (EPS) para que os enfermeiros se apropriem de seu trabalho clínico na perspectiva da clínica ampliada.Nestes termos, Integralidade pressupõe o conceito de cuidado em saúde, a ser trabalhado e desenvolvido continuamente em busca de uma assistência cada vez mais efetiva, global e completa para o indivíduo, família e comunidade. 
O tema "Papel da atenção básica", abordado por apenas três sujeitos, revela visão holística, integrar serviços para atender os usuários. Nota-se que há vários atributos da atenção básica que não foram mencionados pelos sujeitos. Os sujeitos expressam:

“(...) a integralidade (...) é a função da atenção básica (...) descobrir vários pontos da história dele para fazer intervenções. (II,1) (...) função da atenção básica (...) é resolver e ver ele como um todo (...)" (VI, 2).

Nessa perspectiva, nota-se a necessidade de fomentar esta temática dos atributos da atenção básica relacionando com a integralidade por meio de intervenções educativas para os enfermeiros.

O tema "Dimensões da Integralidade", abordado por apenas cinco sujeitos satisfaz o conjunto de cuidados demandados por um indivíduo que procura a atenção básica proposta pelo SUS, conforme o referencial teórico deste estudo. Conforme explicitados abaixo pelos sujeitos entrevistados: Com o atendimento integral, o indivíduo acessa a unidade e a gente consegue atender ele em todos os níveis: bio, físico, psicossocial.

“(...) É importante o diálogo, discussões, debates reuniões, traçar metas a partir dos problemas identificados (...)" (XXI-2).

Outro ângulo de aproximação à Integralidade considera as redes de serviços em distintos níveis de complexidade e de competências. Os sujeitos abaixo expressam esta visão da seguinte forma:

“(...) consegue ver (...) a questão de prevenção, promoção, tratamento, reabilitação (...) (XVIII-2). A integralidade (...) é promoção e prevenção da saúde pro usuário, dar continuidade ao tratamento deste paciente (...)" (XX,1).

Os sujeitos acima reconhecem a integralidade no cuidado de pessoas, grupos e coletividade como um eixo organizativo de ações em saúde para atender as necessidades de saúde das populações, como garantia do acesso aos níveis de atenção à saúde.

Os relatos da maioria dos sujeitos trazem o tema "Desafios para a integralidade" em diversos aspectos como fragmentação da assistência, modelo queixa-conduta e médico centrado, falta de comunicação entre os serviços, alta demanda de serviços, falta de treinamento dos profissionais.

"(...) a assistência é fragmentada tanto na área médica como na área enfermagem (I-2) (...) a população está acostumada com o método (...) médicocêntrico (...) queixa e conduta" (II-2).

Os sujeitos entrevistados em sua maioria revelaram a dificuldade de envolver o médico no trabalho em equipe. Facchini LA, et al. (2018) traz os valores da base interdisciplinar, intersetorial são contrários ao modelo biomédico que fica enfraquecido com a valorização da interdisciplinaridade nas relações de equipe.

"A integralidade não foi assumida por todos, ela não é vista, por falta da educação permanente (...) (V-III). (...) Esta comunicação com as redes de atenção (...) a falta de conversa entre os serviços (...) é o mais difícil (...)” (VII-2)

Apesar das diretrizes vigentes, muitos processos de trabalhos permanecem ainda na prática de um modelo médico centrado, fragmentado e curativo. A implementação da integralidade é um dos maiores desafios do setor saúde no Brasil por acarretar uma transformação do modelo assistencial.

"O trabalho em equipe é muito importante (...) acredito que a formação profissional (...) as capacitações frequentes (...) são importantes (...) surgem protocolos novos (...) tem que ta se atualizando (...)" (XII-3).

Corroborando com pesquisas, Costa NR (2016) traz que os sujeitos compreendem a organização do trabalho e a resolubilidade com enfoque no trabalhador/equipe. Enfatiza-se, assim, a importância da interação da enfermeira para integralidade e organização do trabalho em equipe. Assim, a equipe quando se torna interdisciplinar passa vivenciar o trabalho em saúde com a participação de todos no planejamento, implementação e avaliação integral da assistência. 
O tema "Papel da atenção básica", abordado por apenas três sujeitos, revela visão holística, integrar serviços para atender os usuários. Nota-se que há vários atributos da atenção básica que não foram mencionados pelos sujeitos. Os sujeitos expressam:

"(...) a integralidade (...) é a função da atenção básica (...) descobrir vários pontos da história dele para fazer intervenções." (II,1)

\section{“(...) função da atenção básica (...) é resolver e ver ele como um todo (...)” (VI,2).}

Rangel RF, et al. (2019) comenta que a atenção básica pode desenvolver capacidade de escuta e de formação de vínculos positivos, que considerem a singularidade de cada usuário; a resolutividade clínica; o suporte técnico-pedagógico para os profissionais; o acesso a recursos situados fora da $A B$; os modos de organização do processo de trabalho e gestão das agendas. Além disso, para Geremia DS (2020), a integralidade pode ser buscada na atenção básica quando profissionais visam o apoio dos trabalhadores de outros setores (intersetorialidade) para dar suporte em questões que somente a unidade de saúde não é suficiente para resolução, sendo uma ação integrada dos diversos setores mais eficazes. Nessa perspectiva, nota-se a necessidade de fomentar esta temática dos atributos da atenção básica relacionando com a integralidade por meio de intervenções educativas para os enfermeiros.

O tema "Dimensões da Integralidade", abordado por apenas cinco sujeitos satisfaz o conjunto de cuidados demandados por um indivíduo que procura a atenção básica proposta pelo SUS, conforme o referencial teórico deste estudo.

Para Loch-Neckel G, et al. (2009), o centro das atenções dos profissionais de saúde é o sujeito que necessita de cuidados no seu âmbito físico, mental, social, espiritual, e outros capazes de atender as suas expectativas de saúde. Conforme explicitados abaixo pelos sujeitos entrevistados: Com o atendimento integral, o indivíduo acessa a unidade e a gente consegue atender ele em todos os níveis: bio, físico, psicossocial.

“(...) É importante o diálogo, discussões, debates reuniões, traçar metas a partir dos problemas identificados (...) "(XXI-2).

"(...) a gente tem aquela assistência a criança, ao adolescente, a mulher, ao homem (...) ao idoso" (XVIII-2).

Outro ângulo de aproximação à Integralidade considera as redes de serviços em distintos níveis de complexidade e de competências. Os sujeitos abaixo expressam esta visão da seguinte forma:

“(...) consegue ver (...) a questão de prevenção, promoção, tratamento, reabilitação (...) (XVIII-2). A integralidade (...) é promoção e prevenção da saúde pro usuário, dar continuidade ao tratamento deste paciente (...)" (XX,1).

Os sujeitos acima reconhecem a integralidade no cuidado de pessoas, grupos e coletividade como um eixo organizativo de ações em saúde para atender as necessidades de saúde das populações, como garantia do acesso aos níveis de atenção à saúde. Constatamos que as dimensões da integralidade foram apontadas por apenas cinco sujeitos entrevistados. O que nos leva a refletir sobre a qualidade da assistência prestada aos usuários que procuram os serviços públicos de saúde. Os relatos da maioria dos sujeitos trazem o tema "Desafios para a integralidade" em diversos aspectos como fragmentação da assistência, modelo queixaconduta e médico centrado, falta de comunicação entre os serviços, alta demanda de serviços, falta de treinamento dos profissionais.

“(...) a assistência é fragmentada tanto na área médica como na área enfermagem” (I-2).

"(...) dificuldade seria o número aumentado de demanda (...) a população está acostumada com o método (...) médicocêntrico (...) queixa e conduta” (II-2).

Os sujeitos entrevistados em sua maioria revelaram a dificuldade de envolver o médico no trabalho em equipe, além da população estar acostumada com o modelo centrado no profissional médico valorizando pouco o trabalho da equipe de saúde, além da forte fragmentação da assistência em saúde. 
Rover MRM, et al. (2016) aponta quea fragmentação do cuidado vem sendo refletida e tem se caminhado para a organização interdisciplinar do trabalho na saúde valorizando todos os integrantes da equipe. Yamamoto TS, (2016) traz que os valores da base interdisciplinar, intersetorial são contrários ao modelo biomédico que fica enfraquecido com a valorização da interdisciplinaridade nas relações de equipe. Kalichman AO e Ayres JRCM (2016) refletem que ao atuarem separadamente, os diversos profissionais perdem a chance de se integrar uns com os outros, o que poderia aprimorar a qualidade da assistência e a interação com os usuários, fortalecendo a integralidade e tornando o trabalho mais prazeroso e motivador. A integralidade não foi assumida por todos, ela não é vista, por falta da educação permanente.

"(...) (V-III). (...) Esta comunicação com as redes de atenção (...) a falta de conversa entre os serviços (...) é o mais difícil(...)" (VII-2).

Apesar das diretrizes vigentes, muitos processos de trabalhos permanecem ainda na prática de um modelo médico centrado, fragmentado e curativo. A implementação da integralidade é um dos maiores desafios do setor saúde no Brasil por acarretar uma transformação do modelo assistencial.

Mendes EV (2012) traz que a formação acadêmica também é um fator marcante na fragmentação das práticas, enfatizado pela dificuldade dos profissionais de desenvolverem o trabalho em equipe. Apesar dos desafios já explicitados, os relatos da maioria dos sujeitos trazem também o tema "Fortalezas para a integralidade" em diversos aspectos como trabalho em equipe, intersetorialidade, equipes completas, educação permanente em saúde, integração com o NASF (Núcleo de Apoio à Saúde da Família), olhar ampliado para o usuário e seu contexto.

"(...) integralidade é (...) pensar (...) na saúde da família (...) nos princípios do SUS (...) (XII-1). O trabalho em equipe é muito importante (...) acredito que a formação profissional (...) as capacitações frequentes (...) são importantes (...) surgem protocolos novos (...) tem que ta se atualizando (...)" (XII-3).

Corroborando com estudos de Andrade SR (2013) os sujeitos compreendem a organização do trabalho e a resolubilidade com enfoque no trabalhador/equipe. Enfatiza-se, assim, a importância da interação da enfermeira para integralidade e resolubilidade da assistência, eficiência e organização do trabalho em equipe e satisfação profissional.

O trabalho em equipe foi uma fortaleza proposta aos profissionais da atenção básica. Dentro de seus processos de trabalho individuais, eles descobriram a importância de se fazer saúde de uma forma integral. Outro ponto de vista acerca da integralidade é ser indispensável o envolvimento das redes de cuidado e do trabalho com os pares - os serviços, o ensino e a comunidade.

Silva MIA, et al. (2019), relata que a interdisciplinaridade também revelada pelos sujeitos é uma estratégia para reduzir a fragmentação do cuidado fortalecendo a integralidade reorganizando o processo de trabalho com objetivo de atender a complexidade das necessidades da população. Assim, a equipe quando se torna interdisciplinar passa vivenciar o trabalho em saúde com a participação de todos no planejamento, implementação e avaliação integral da assistência.

Os sujeitos do estudo valorizam a EPS. E ao refletir sobre a Política Nacional de Educação Permanente em Saúde nota-se que ela aparece como uma proposta de ação estratégica para contribuir para a transformação e a qualificação das práticas de saúde, a organização das ações e dos serviços, dos processos formativos e das práticas pedagógicas na formação e no desenvolvimento dos trabalhadores de saúde.

\section{CONCLUSÃO}

O presente estudo permitiu reflexões acerca da integralidade, todos os profissionais devem se conscientizar que a atenção à saúde deve ser acolhedora, humanizada, contextualizada pelo olhar para o território. A integralidade acontece como fruto de uma construção coletiva, o que envolve o trabalho em equipe por meio de uma boa articulação das práticas intersetoriais, a própria prática do acolhimento por meio da escuta qualificada e atender as necessidades de saúde trazidas por cada um. É necessária a organização dos serviços de saúde estreitando os laços entre os serviços de saúde como forma de garantir a continuidade da assistência, o diálogo, o estabelecimento de fluxos de atendimento entre os serviços, o próprio uso das ferramentas de comunicação como telefone, e-mail podem estreitar o vínculo entre os serviços. 


\section{REFERÊNCIAS}

1. BRASIL. Constituição da República Federativa do Brasil: promulgada em 5 de outubro de 1988. Brasília: Senado Federal; 1988.

2. BRASIL. Lei no 8.080 , de 19 de setembro de 1990. Lei Orgânica da Saúde Institui o Sistema Único de Saúde. Diário Oficial da República Federativa do Brasil ;1990; 20 set.

3. BRASIL. Ministério da Saúde (BR). Portaria n. 648, de 28 de março de 2006.Política Nacional de Atenção Básica. Diário Oficial da União, Brasília (DF); 2006.

4. BOUSQUAT A, et al. A atenção primária em regiões de saúde: política, estrutura e organização. Cad. Saúde Pública,2019; 35( 2): e00099118.

5. COSTA, NR. A Estratégia de Saúde da Família, a atenção primária e o desafio das metrópoles brasileiras. Ciênc. saúde coletiva, Rio de Janeiro,2016; 21(5): 1389-1398.

6. FACCHINI LA, et al. Qualidade da Atenção Primária à Saúde no Brasil: avanços, desafios e perspectivas. Saúde debate,2018; 42(1): 208-223.

7. GOMIDE MFS. A satisfação do usuário com a atenção primária à saúde: uma análise do acesso e acolhimento. Interface (Botucatu), 2018; 22(65): 387-398.

8. HINO P, et al. Integralidade na perspectiva da saúde coletiva: caminhos para a formação do enfermeiro. Rev. Bras. Enferm. 2019; 72(4): 1119-1123.

9. LANZONI GMM, MEIRELLES BHS. Liderança do enfermeiro: elemento interveniente na rede de relações do agente comunitário de saúde. Rev. bras. enferm., Brasília, 2013; 66(4).

10. LIMA SGG, et al. O processo de incorporação de tecnologias em saúde no Brasil em uma perspectiva internacional. Ciência \& Saúde Coletiva, 2019; 24: 1709-1722.

11. MAGALHAES MGM, ALVIN, NAT. Práticas integrativas e complementares no cuidado de enfermagem: um enfoque ético. Esc Anna Nery (impr.),2013; 17(4): 646-653.

12. MARTINS J, BICUDO MAV. A pesquisa qualitativa em psicologia: fundamentos e recursos básicos. $2^{a}$ ed. São Paulo (SP): Moraes; 1994:44.

13. MEDEIROS AC, et al. Comprehensiveness and humanization of nursing care management in the Intensive Care Unit. Rev Esc Enferm USP,2016; 50(5): 816-822.

14. MENDES EV. As redes de atenção à saúde. Ciênc. saúde coletiva, Rio de Janeiro,2010; 15 (5): 2297-2305.

15. MERLEAU PM. Fenomenologia da percepção. São Paulo: Martins Fontes, 2006.

16. SADALA MLA. A fenomenologia como método para investigar a experiência vivida: uma perspectiva do pensamento de Husserl e de Merleau-Ponty. Seminário Internacional de Pesquisa e estudos qualitativos, 2004.

17. OLIVEIRA IC, et al. Integralidade: Algumas Reflexões. Revista Brasileira de Educação Médica, 2018; 42(3): $146-152$.

18. SILVA MVS, et al. Sentidos atribuídos à integralidade: entre o que é preconizado e vivido na equipe multidisciplinar. Interface (Botucatu), Botucatu, 2017; 21 (62): 589-99.

19. YAMAMOTO TS, et al. Educação Permanente em Saúde como prática avaliativa amistosa à integralidade em Teresópolis, Rio de Janeiro. Trab. educ. saúde, Rio de Janeiro, 2015; 13( 3): 617-38.

20. RANGEL RF, et al. Concepções de docentes de enfermagem sobre integralidade. Revista da Rede de Enfermagem do Nordeste-Rev Rene, 2012; 13(3).

21. GEREMIA DS. Atenção Primária à Saúde em alerta: desafios da continuidade do modelo assistencial. Physis, Rio de Janeiro, 2020; 30 (1): e300100.

22. LOCH-NECKEL G, et al. Desafios para a ação interdisciplinar na atenção básica: implicações relativas à composição das equipes de saúde da família. Ciênc Saúde Coletiva, 2009; 14 (1): 1463-72.

23. LOUVISON MCP. Regionalização dos sistemas de saúde como resposta às desigualdades territoriais: um debate necessário. Cadernos de Saúde Pública, 2019; 35 (2): e00116019. Disponível em: <https://doi.org/10.1590/0102311X00116019>

24. ROVER MRM, et al. Da organização do sistema à fragmentação do cuidado: a percepção de usuários, médicos e farmacêuticos sobre o Componente Especializado da Assistência Farmacêutica. Physis, Rio de Janeiro, 2016; 26 (2):691-711.

25. KALICHMAN AO, AYRES JRCM. Integralidade e tecnologias de atenção à saúde: uma narrativa sobre contribuições conceituais à construção do princípio da integralidade no SUS. Cad. Saúde Pública, Rio de Janeiro, 2016; 32(8).

26. MENDES EV. A APS no Brasil. In: Mendes EV, organizador. O cuidado das condições crônicas na atenção primária à saúde: o imperativo da consolidação da estratégia da saúde da família. Brasília: Organização Pan-Americana da Saúde; 2012: 71-99.

27. ANDRADE SR. Melhores práticas na atenção básica a saúde e os sentidos da integralidade. Esc. Anna Nery, Rio de Janeiro, 2013; 17 (4).

28. SILVA MIA, et al. Educação Permanente em Saúde na Estratégia de Saúde da Família: Relato da Elaboração do Plano de Intervenção. Revista Eletrônica Acervo Saúde, 2019; 11(16): e1527. 